\title{
Synthesis of Hexagonal Boron Carbonitride without Nitrogen Void Defects
}

\author{
Md. Abdul Mannan1*, Yuji Baba ${ }^{2,3}$, Tetsuya Kida4, Masamitsu Nagano4, Hideyuki Noguchi4 \\ ${ }^{1}$ Department of Chemistry, University of Rajshahi, Rajshahi, Bangladesh \\ ${ }^{2}$ Synchrotron Radiation Research Unit, Quantum Beam Science Directorate, Japan Atomic Energy Agency, \\ Ibaraki-ken, Japan \\ ${ }^{3}$ Sector of Fukushima Research and Development, Japan Atomic Energy Agency, Fukushima-shi, Japan \\ ${ }^{4}$ Department of Chemistry and Applied Chemistry, Saga University, Honjyo, Japan \\ Email: *
}

Received 17 January 2015; accepted 2 May 2015; published 7 May 2015

Copyright (C) 2015 by authors and Scientific Research Publishing Inc.

This work is licensed under the Creative Commons Attribution International License (CC BY).

http://creativecommons.org/licenses/by/4.0/

(c) (i) Open Access

\begin{abstract}
The synthesis and structure of hexagonal boron carbonitride ( $h$-BCN) film on polycrystalline diamond surface were reported. Polycrystalline diamond and/or diamond-like carbon were first fabricated on Si (100) and then diamond like carbon was used as substrate. The deposition was performed by radio frequency plasma enhanced chemical vapor deposition. In order to reduce the content of nitrogen void defects, the deposition was performed at the high temperature of $950^{\circ} \mathrm{C}$ under the working pressure of $2.6 \mathrm{~Pa}$. The typical sample with atomic composition of $\mathrm{B}_{31} \mathrm{C}_{37} \mathrm{~N}_{26} \mathrm{O}_{6}$ in the $h$-BCN lattice was characterized by X-ray photoelectron spectroscopy. The fine structure of the film was studied by near-edge X-ray absorption fine structure (NEXAFS) measurements. The B $K$-edge and $N K$-edge of NEXAFS spectra revealed that the synthesized $h$-BCN film had the ideal honeycomb-like $\mathrm{BN}_{3}$ configuration without nitrogen void defects.
\end{abstract}

\section{Keywords}

h-BCN, Defect, Chemical Vapor Deposition, XPS, NEXAFS

\section{Introduction}

Hexagonal boron carbonitride ( $h$-BCN) compound has been focusing as essential nanomaterials for its various applications especially in nanoelectronics due to its semiconducting property. The $h$-BCN could be used in photoluminescent devices, solar cells, sensor, high temperature nanotransistor, lightweight electrical conductors, or

${ }^{*}$ Corresponding author.

How to cite this paper: Mannan, Md.A., Baba, Y., Kida, T., Nagano, M. and Noguchi, H. (2015) Synthesis of Hexagonal Boron Carbonitride without Nitrogen Void Defects. Materials Sciences and Applications, 6, 353-359.

http://dx.doi.org/10.4236/msa.2015.65041 
high temperature lubricants etc. [1] [2]. The efforts of preparing $h$-BCN have been initiated since 1980s [3]-[6], and till to date numerous efforts have been aimed to synthesize single phase of $h$-BCN using different methods [7]-[11]. However, most of those compounds are amorphous and/or mixtures of pure BN and graphite domains. In fact, the most challenging obstacle for preparing crystalline $h-\mathrm{BCN}$ is phase separation and presence of different defect fine structures. Indeed, single phase of crystalline $h$-BCN with nitrogen void defect free structure is not achieved yet. For the synthesis of $h$-BCN film, a plasma enhanced chemical vapor deposition (PE-CVD) method is suitable, since the PE-CVD provides unique advantages over other techniques such as freedom to choose the substrate material and a uniform deposition over relatively large area, and controllable deposition parameter to prepare BCN of various compositions and thickness. Furthermore, plasma plays a very active role for film deposition because it makes ions and radicals.

In the previous paper, we report that we have successfully synthesized $h$-BCN on Si (100) [12] [13]. The $h$-BCN basal planes perpendicularly standing up orientation with respect to silicon surface were obtained. Also we synthesized $h$-BCN on $\mathrm{Ni}$ (111) and polycrystalline Ti. Randomly oriented $h$-BCN films were found with respect to nickel and titanium substrates. However, the $h$-BCN films deposited on these substrates have some nitrogen void defects, which were revealed by the measurements of the near-edge X-ray absorption fine structure (NEXAFS) [12]-[14]. In order to reduce the number of nitrogen void defects in $h$-BCN, we consider that it would be better to apply higher temperature during the deposition. Also it is better to use a substrate with similar lattice constant with that of $h$-BCN. In this paper, our succeeding research to obtain $h$-BCN without nitrogen void defects by applying high temperature in choosing diamond as substrate is presented.

\section{Experimental Method}

Hexagonal boron carbonitride ( $h$-BCN) films were synthesized on polycrystalline diamond surface by radio frequency plasma enhanced chemical vapor deposition (RF-PECVD) using tris-dimethylaminoborane (TDMAB) as precursor. The experimental method is similar as we reported in the reference [12]. In this study, diamond and/or diamond-like carbon were first developed on $\mathrm{Si}(100)$ wafer and then it was used as substrate. The wafer $\left(1 \times 1 \mathrm{~cm}^{2}\right)$ was cleaned by ultrasonic cleaner in acetone bath followed by hydrofluoric acid $(50 \%)$ then the ascleaned wafer was etched with diamond powder in ethanol for 25 min. After etching, the wafer was washed three times in acetone followed by in ethanol. X-ray diffraction (XRD) (Rigaku diffractometer) using $\mathrm{CuK}_{\alpha}$ radiation (30 kV, $20 \mathrm{~mA}$ ) was taken for the as-cleaned wafer in order to confirm nonappearance of diamond powder on the wafer surface. Then the wafer was installed in the reaction chamber where the inner pressure was set at $\sim 2.6 \mathrm{~Pa}$. The substrate was heated by RF plasma at around $950^{\circ} \mathrm{C}$ and then $\mathrm{CH}_{4}$ plasma was flown into the chamber for $30 \mathrm{~min}$ at flow rate of $10 \mathrm{sccm}$. The XRD confirmed that diamond and/or diamond-like carbon were formed on the wafer due to the flounce of $\mathrm{CH}_{4}$ plasma. Finally, BCN films were synthesized in-situ by the flounce of plasma of TDMAB on the diamond surface.

The synthesis was done with varying temperature from $950^{\circ} \mathrm{C}$ to $1050^{\circ} \mathrm{C}$. Since the films compositions were almost identical, only one typical result has been discussed. The films were characterized by X-ray photoelectron spectroscopy (XPS), near-edge X-ray absorption fine structure (NEXAFS) and field emission scanning electron microscopy (FE-SEM) (JSM-6700 FSS, JEOL), respectively. The XPS and NEXAFS measurements were performed at the Beam Line 27A and the Beam Line 11A of the KEK photon factory, Tsukuba, Japan. The XP spectra were taken by using synchrotron radiation of energy $\mathrm{h} v=2.2 \mathrm{keV}$. The NEXAFS spectra were measured using $300 \mathrm{l} / \mathrm{mm}$ laminar gratings in an UHV chamber $\left(\sim 1.7 \times 10^{-6} \mathrm{~Pa}\right)$ with total electron yield mode by recording sample current. Details of XPS and NEXAFS have been described here [15].

\section{Results and Discussion}

Figure 1 show high resolution FE-SEM surface morphologies for the typical sample prepared on polycrystalline diamond surface. It can be seen that the crystals are almost homogeneously distributed at micrometer size all over the surface. High-magnification image (Figure 1(b)) also confirms the homogeneity of the crystals. However, FE-SEM image presents only surface morphologies in micrometer scale. Therefore, composition and structures are studied by spectroscopic methods.

Figure 2 shows XRD patterns for the typical BCN sample on diamond surface (top), diamond film (middle) and silicon wafer (bottom). The XRD patterns confirm nonexistence of diamond powder on the silicon wafer (bottom). The pattern for the as-deposited diamond film (middle) shows a prominent peak at $2 \theta=44.0^{\circ}$ which 


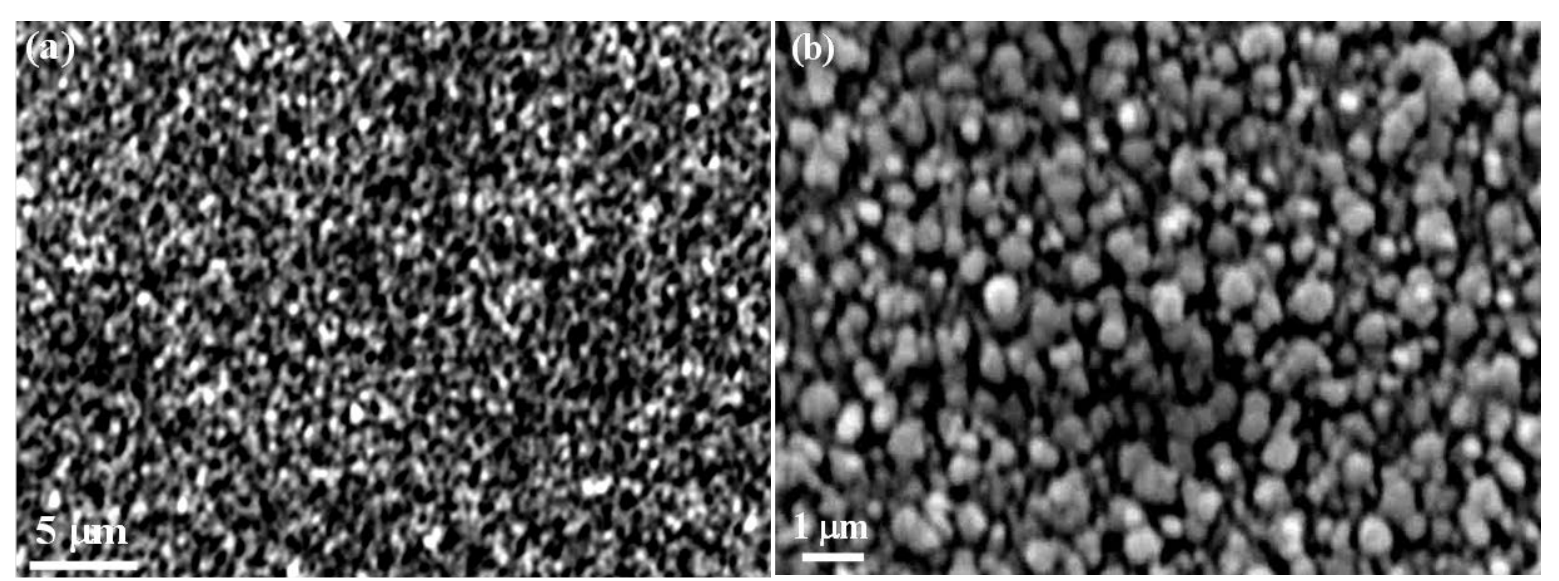

Figure 1. FE-SEM image: (a) Low-magnification; (b) High-magnification of the typical sample on diamond surface.

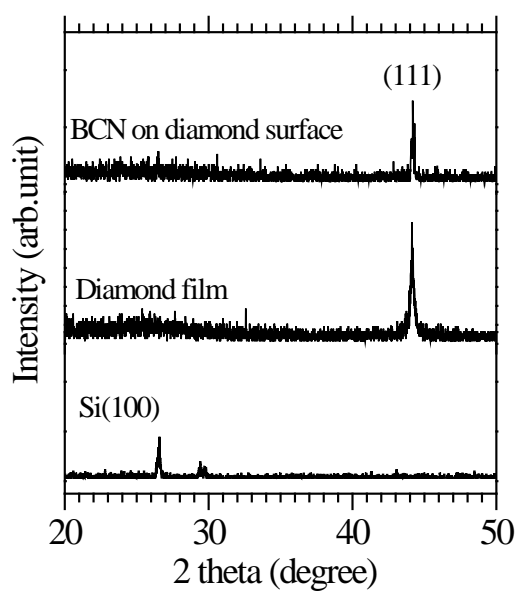

Figure 2. XRD pattern of typical sample (top), as-developed diamond (middle) and silicon wafer after etching with diamond powder (bottom).

corresponds to the diffraction from the (111) plane in the diamond lattice [16]. It is clear from the figure that the diffraction peak corresponding to diamond phase or diamond-like carbon phase has been developed on the silicon wafer. The XRD pattern for as-deposited BCN film also shows a single peak at $2 \theta=44.0^{\circ}$ with low intensity. But no peak observed at $2 \theta=26.3^{\circ}$ in the pattern which previously suggested in formation of $h$-BCN lattice in the (002) plane [13] [17]. No peaks corresponding to development of other phases like graphite or amorphous carbon are observed. The peak at $2 \theta=44.0^{\circ}$ for the sample pattern might be due to development of diamond on the wafer or it may come from the as-developed diamond film. From XRD analyses we could not really say the growth of the crystalline $h$-BCN phase due to the short-rang atomic order of the lattice.

Figure 3 shows wide scan XP spectrum of the typical sample shown in Figure 2. The composition $B_{31} C_{37} N_{26} \mathrm{O}_{6}$ shown inside of the figure was estimated from $\mathrm{I}_{\mathrm{B} 1 \mathrm{~s}} / \sigma_{\mathrm{B} 1 \mathrm{~s}}: \mathrm{I}_{\mathrm{C} 1 \mathrm{~s}} / \sigma_{\mathrm{C} 1 \mathrm{~s}}: \mathrm{I}_{\mathrm{N} 1 \mathrm{~s}} / \sigma_{\mathrm{N} 1 \mathrm{~s}}$, where I is intensity of photoelectron peak and $\sigma$ is photoionization cross-section for the respective core-level indicated as subscript. From the wide scan XPS results it is confirmed that the BCN film is composed of B, C, and N atoms. The O1s peak corresponding to oxygen was found due to the surface contamination. However, the oxygen contamination does not affect the bulk structure of the BCN film, because the formation of the B-O bond was not observed in the B K-edge NEXAFS spectra which probe deeper region than that of XPS (discussed later). In order to confirm that the sample was really made up of B-C-N atomic hybrid rather than a mixture of BN and graphite binary phases, narrow scan B1s, C1s and N1s XPS spectra were measured.

Figure 4 shows the B1s, C1s and N1s narrow scan XPS spectra for the typical sample. It is seen that all the B1s (190.8 eV), C1s (284.5 eV) and N1s (398.0 eV) spectra found almost symmetrical, non-splitting and single Gaussian-like curve. Peak splitting around $192.0 \mathrm{eV}$ in the B1s spectra, $287.5 \mathrm{eV}$ in the C1s spectra and 401.0 


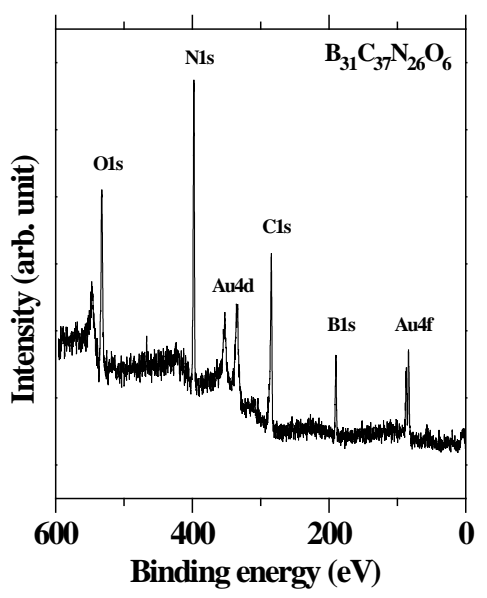

Figure 3. XPS wide scan spectrum for the sample shown in Figure 2. The sample surface was irradiated with the SR beam of $2.20 \mathrm{keV}$ photons with the step energy of $0.22 \mathrm{eV}$.

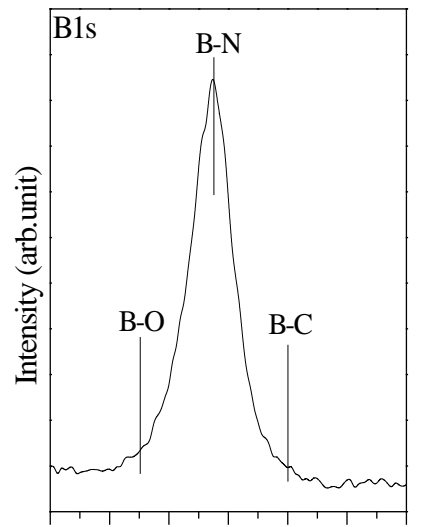

196194192190188186184

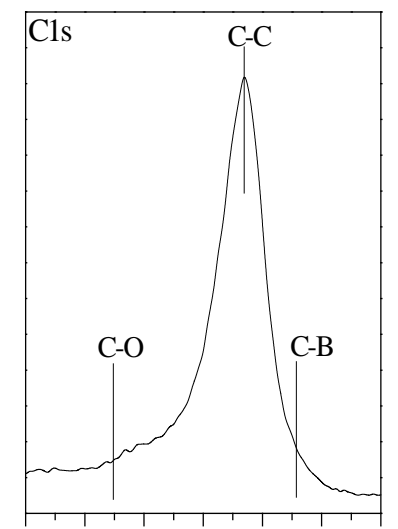

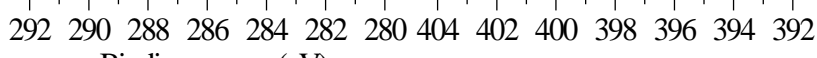

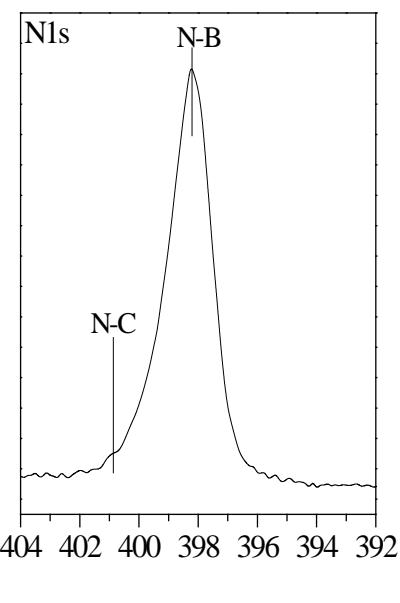

Binding energy $(\mathrm{eV})$

Figure 4. B1s, C1s and N1s narrow scan XPS spectra for the typical sample shown in Figure 2.

$\mathrm{eV}$ in the N1s spectra would observed if there is an existence of formation of BN-like and graphite-like phases as observed in the references [10] [18]. Therefore, formation of separated binary phases such as BN and/or graphite was not obvious in the present sample. NEXAFS measurements were performed for further assessment of the nitrogen void fine structures.

Figure 5 shows B $K$-edge $\pi^{*}$ resonance NEXAFS spectra taken at normal $\left(\theta=90^{\circ}\right)$ and at grazing $\left(\theta=15^{\circ}\right)$ incidence angles of the X-ray for the typical sample. Since the intensity and shape of the $\sigma^{*}$ resonance peak was unchanged with grazing incidence and hence less informative. Therefore, only $\pi^{*}$ resonance peak has been considered because several fine structures usually found when the spectra were taken at grazing incidence angle within the $\pi^{*}$ resonance area. It is seen that a single Gaussian-like peak $\mathrm{B}_{2}$ has been observed for the sample at photon energy around $192.1 \mathrm{eV}$ and intensity of the peak does not change at the grazing $\left(\theta=15^{\circ}\right)$ incidence, suggesting factual formation of hexagonal $h$-BCN hybrids with a similar configuration like- $\mathrm{BN}_{3}$ in the honeycomb-like structure.

It has been reported that if a boron atom is bonded with three carbon atoms forming $\mathrm{BC}_{3}$-like configuration, the $\pi^{*}$ resonance peak would appear around $191.0 \mathrm{eV}$ (marked $\mathrm{B}_{1}$ in Figure 5(a)) [19], but no peak is seen in this energy region. Also if $\mathrm{B}-\mathrm{O}_{2} \mathrm{C}$ and $\mathrm{B}-\mathrm{ON}_{2}$ bonds are formed, the $\pi^{*}$ resonance peak would appear around $192.5 \mathrm{eV}$ (marked $\mathrm{B}_{3}$ ) and $193.0 \mathrm{eV}$ (marked $\mathrm{B}_{4}$ ), respectively [20] [21], but scarcely any peaks are observed in these energy regions. Probing depth of NEXAFS in total electron yield is in the order of $10 \mathrm{~nm}$ [22], which is fairly deeper than that of XPS. Therefore, it is confirmed that the oxygen contamination observed in the XPS wide scan (Figure 3) does not affect the bulk structure of the BCN film. In order to clarify the existence of the 
void structure, the NEXAFS spectra for BCN on diamond are compared with those for the samples deposited on HOPG and Ni substrates. All the nitrogen void fine structures $B_{1}, B_{3}$ and $B_{4}$ are clearly observed (Figure $5(b)$ and Figure 5(c)). Same findings were reported in the case of silicon and titanium substrates [12] [14].

However, the spectra for the present sample show no fine structures other than the main peak $B_{2}$. The $B$ $K$-edge NEXAFS analyses confirmed that the $h$-BCN layers composed of highly ordered configuration without nitrogen void and/or with few nitrogen void defects structures.

Figure 6 shows $\mathrm{N} K$-edge resonance NEXAFS spectra for the typical sample. It has been observed that the $\pi^{*}$ as well as $\sigma^{*}$ resonance peak of the $\mathrm{N} K$-edge does not change with grazing incidence angles. Moreover, the spectral shape is very similar to each other and not fine structures usually found near $397.5 \mathrm{eV}$ (marked $\mathrm{N}_{1}$ in the Figure 6) due to nitrogen void are appearing [19]. This observation also confirms that the atomic arrangement around $\mathrm{N}$ atoms is very homogenous and similar to each other in comparison with those around the $\mathrm{B}$ atoms suggesting the ideal honeycomb $h$-BCN hybrid configuration.

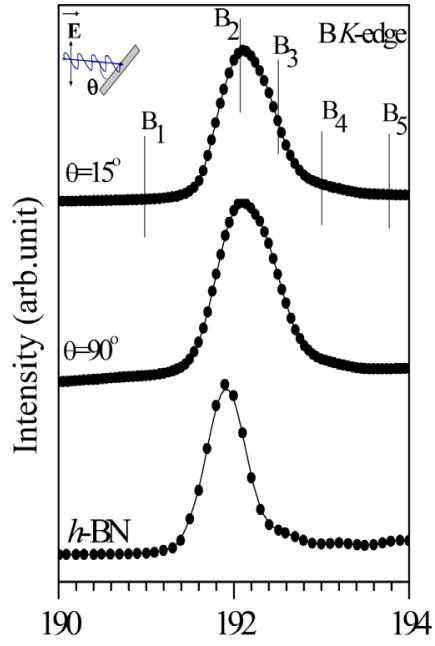

(a)

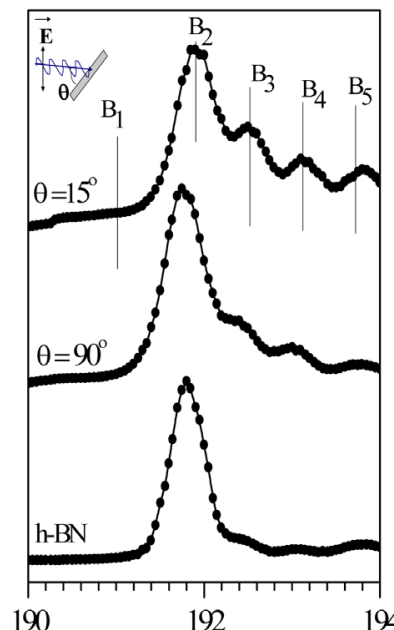

(b)

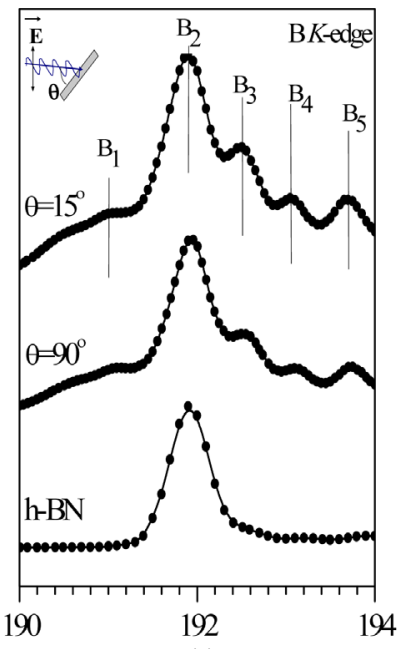

(c)

Photon energy (eV)

Figure 5. The B K-edge NEXAFS spectra: (a) For the typical sample deposited on diamond surface; (b) Sample deposited on HOPG substrate [15]; (c) Sample deposited on Ni substrate [14]. The spectra were recorded at step energy of $0.05 \mathrm{eV}$ with normal $\left(\theta=90^{\circ}\right)$ and at grazing incidence angles $(\theta$ $=15^{\circ}$ ). Definition of $\theta$ is shown inside of this figure. The bottom spectrum for pyrolytic $h$-BN is shown as reference.

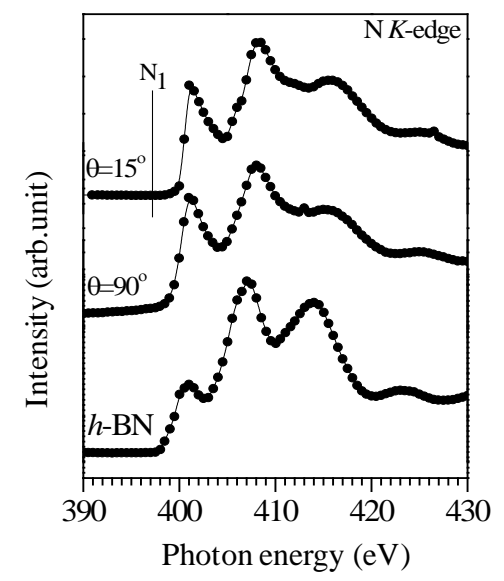

Figure 6. The $\mathrm{N} K$-edge NEXAFS spectra for the typical sample. The spectra were recorded at step energy of $0.05 \mathrm{eV}$ with normal $\left(\theta=90^{\circ}\right)$ and at grazing incidence angles $\left(\theta=15^{\circ}\right)$. Spectrum for pyrolytic $h$-BN is shown as reference. 


\section{Conclusion}

We synthesized nano-crystalline hexagonal BCN films on polycrystalline diamond surface by RF-PECVD using TDMAB. The crystalline phase could not be detected by XRD due to short-range atomic order of the (002) lattice plane. However, the crystalline phase in the sample is confirmed by NEXAFS analyses. XPS suggests that the film is composed of B-C-N atomic hybrids. It is suggested from the B K-edge and N K-edge NEXAFS spectral analyses that the hexagonal honeycomb $h$-BCN lattice has an ideal structure without nitrogen void defects.

\section{Acknowledgements}

The authors are grateful to the staff of the KEK-PF to run the experiments (Proposal No. 2008G502).

\section{References}

[1] Yu, J., Ahn, J., Yoon, S.F., Zhang, Q., Rusli, B.G., Chew, K., Yu, M.B., Bai, X.D. and Wang, E.G. (2000) Semiconducting Boron Carbonitride Nanostructures: Nanotubes and Nanofibers. Applied Physics Letters, 77, 1949-1951. http://dx.doi.org/10.1063/1.1311953

[2] Zhi, C.Y., Guo, J.D., Bai, X.D. and Wang, E.G. (2002) Adjustable Boron Carbonitride Nanotubes. Journal of Applied Physics, 91, 5325-5333. http://dx.doi.org/10.1063/1.1459596

[3] Montasser, K., Hattori, S. and Monita, S. (1984) Transparent B-C-N-H Thin Films Formed by Plasma Chemical Vapour Deposition. Thin Solid Films, 117, 311-317. http://dx.doi.org/10.1016/0040-6090(84)90361-4

[4] Maya, L. (1988) Aminoborane Polymers as Precursors of C-N-B Ceramic Materials. Journal of the American Ceramic Society, 71, 1104-1107. http://dx.doi.org/10.1111/j.1151-2916.1988.tb05799.x

[5] Kouvetaksi, J., Sasaki, T., Shen, C., Hagiwara, R., Lerner, M., Krishnan, K.M. and Bartlett, N. (1989-1990) Novel Aspects of Graphite Intercalation by Fluorine and Fluorides and New B/C, C/N and B/C/N Materials Based on the Graphite Network. Synthetic Metals, 34, 1-7. http://dx.doi.org/10.1016/0379-6779(89)90355-X

[6] Maya, L. and Harris, L.A. (1990) Pyrolytic Deposition of Carbon Films Containing Nitrogen and/or Boron. Journal of the American Ceramic Society, 73, 1912-1916. http://dx.doi.org/10.1111/j.1151-2916.1990.tb05244.x

[7] Zhang, T., Zeng, S.F. and Wen, G. (2014) A Simple Precursor Pyrolysis Rout to BCN Nanoflakes. Materials Letters, 132, 277-280. http://dx.doi.org/10.1016/j.matlet.2014.06.098

[8] Kang, Y., Chu, Z., Zhang, D., Li, G., Jiang, Z., Cheng, H. and Li, X. (2013) Incorporate Boron and Nitrogen into Graphene to Make BCN Hybrid Nanosheets with Enhanced Microwave Absorbing Properties. Carbon, 61, 200-208. http://dx.doi.org/10.1016/j.carbon.2013.04.085

[9] Park, J.K., Lee, J.H., Lee, W.S. and Baik, Y.J. (2013) Effect of Substrate Bias and Hydrogen Addition on the Residual Stress of BCN Film with Hexagonal Structure Prepared by Sputtering of a $\mathrm{B}_{4} \mathrm{C}$ Target with $\mathrm{Ar} / \mathrm{N}_{2}$ Reactive Gas. Thin Solid Films, 549, 276-280. http://dx.doi.org/10.1016/j.tsf.2013.06.041

[10] Zhou, F., Wang, Q., Yue, B., Wu, X., Zhuge, L. and Chen, X. (2013) Mechanical Properties and Bonding Structure of Boron Carbon Nitride Films Synthesized by Dual Ion Beam Sputtering. Materials Chemistry and Physics, 138, 215224. http://dx.doi.org/10.1016/j.matchemphys.2012.11.049

[11] Xu, S., Ma, X., Wen, H., Tang, G. and Li, C. (2014) Effect of Annealing on the Mechanical and Scratch Properties of BCN Films Obtained by Magnetron Sputtering Deposition. Applied Surface Science, 313, 823-827. http://dx.doi.org/10.1016/j.apsusc.2014.06.081

[12] Mannan, M.A., Nagano, M., Kida, T., Hirao, N. and Baba, Y. (2009) Characterization of BCN Films Synthesized by Radiofrequency Plasma Enhanced Chemical Vapor Deposition. Journal of Physics and Chemistry of Solids, 70, 20-25. http://dx.doi.org/10.1016/j.jpcs.2008.08.013

[13] Mannan, M.A., Kida, T., Noguchi, H., Nagano, M., Hirao, N. and Baba, Y. (2009) Atomic Arrangement, Composition and Orientation of Hexagonal BCN Films Synthesized by Radiofrequency Plasma Enhanced CVD. Journal of the Ceramic Society of Japan, 117, 503-507. http://dx.doi.org/10.2109/jcersj2.117.503

[14] Mannan, M.A., Baba, Y., Kida, T., Nagano, M., Shimoyama, I., Hirao, N. and Noguchi, H. (2011) Orientation of B-C-N Hybrid Films Deposited on Ni (111) and Polycrystalline Ti Substrates Explored by X-Ray Absorption Spectroscopy. Thin Solid Films, 519, 1780-1786. http://dx.doi.org/10.1016/j.tsf.2010.09.052

[15] Mannan, M.A., Noguchi, H., Kida, T., Nagano, M., Hirao, N. and Baba, Y. (2010) Growth and Characterization of Stoichiometric BCN Films on Highly Oriented Pyrolytic Graphite by Radiofrequency Plasma Enhanced Chemical Vapor Deposition. Thin Solid Films, 518, 4163-4169. http://dx.doi.org/10.1016/j.tsf.2009.11.086

[16] Dilawar, N., Kapil, R., Vankar, V.D., Avasthi, D.K., Kabiraj, D. and Mehta, G.K. (1997) Radiation Hardness of Polycrystalline Diamond Thin Films Irradiated with $100 \mathrm{MeV}^{7+}$ Ions. Thin Solid Films, 305, 88-94. 
http://dx.doi.org/10.1016/S0040-6090(97)00167-3

[17] Torres, R., Caretti, I., Gago, R., Martín, Z. and Jiménez, I. (2007) Bonding Structure of BCN Nanopowders Prepared by Ball Milling. Diamond and Related Materials, 16, 1450-1454. http://dx.doi.org/10.1016/j.diamond.2007.01.009

[18] Bengu, E., Genisel, M.F., Gulseren, O. and Ovali, R. (2009) Theoretical and Spectroscopic Investigations on the Structure and Bonding in B-C-N Thin Films. Thin Solid Films, 518, 1459-1464. http://dx.doi.org/10.1016/j.tsf.2009.09.106

[19] Gago, R., Jiménez, I., Garcia, I. and Albella, J.M. (2002) Growth and Characterization of Boron-Carbon-Nitrogen Coatings Obtained by Ion Beam Assisted Evaporation. Vacuum, 64, 199-204. http://dx.doi.org/10.1016/S0042-207X(01)00314-1

[20] Caretti, I. and Jeménez, I. (2011) Point Defects in Hexagonal BN, BC 3 and $\mathrm{BC}_{\mathrm{x}} \mathrm{N}$ Compounds Studied by X-Ray Absorption Near-Edge Structure. Journal of Applied Physics, 110, Article ID: 023511. http://dx.doi.org/10.1063/1.3602996

[21] Caretti, I. and Jiménez, I. (2011) Composition and Bonding Structure of Boron Nitride $\mathrm{B}_{1-\chi} \mathrm{N}_{x}$ Thin Films Grown by Ion-Beam Assisted Evaporation. Chemical Physics Letters, 511, 235-240. http://dx.doi.org/10.1016/j.cplett.2011.06.001

[22] Frazer, B.H., Gilbert, B., Sonderegger, B.R. and Stasio, G.D. (2003) The Probing Depth of Total Electron Yield in the Sub-KeV Range: TEY-XAS and X-PEEM. Surface Science, 537, 161-167. http://dx.doi.org/10.1016/S0039-6028(03)00613-7 